\title{
Plasmodium falciparum malaria co-infection with tick-borne relapsing fever in Dakar
}

Mamadou A. Diallo ${ }^{1 *}$, Baidy S. Kane ${ }^{2}$, Mouhamadou Ndiaye ${ }^{1}$, Mouhamed Dieng ${ }^{2}$, Khadim Diongue ${ }^{1}$, Aida S. Badiane ${ }^{1}$, Mame Cheikh Seck ${ }^{1}$ and Daouda Ndiaye ${ }^{1}$

\begin{abstract}
Background: West African tick-borne relapsing fever (TBRF) due to Borrelia crocidurae and malaria are co-endemics in Senegal. Although expected to be high, co-infections are rarely reported. A case of falciparum malaria and B. crocidurae co-infection in a patient from Velingara (South of Senegal) is discussed.

Case: A 28 year-old-male patient presented to Aristide Le Dantec Hospital for recurrent fever. He initially presented to a local post health of Pikine (sub-urban of Dakar) and was diagnosed for malaria on the basis of positive malaria rapid diagnostic test (RDT) specific to Plamodium falciparum. The patient was treated as uncomplicated falciparum malaria. Four days after admission the patient was referred to Le Dantec Hospital. He presented with fever $\left(39^{\circ} \mathrm{C}\right)$, soreness, headache and vomiting. The blood pressure was 120/80 mmHg. The rest of the examination was normal. A thick film from peripheral blood was performed and addressed to the parasitology laboratory of the hospital. Thick film was stained with $10 \%$ Giemsa. Trophozoite of P. falciparum was identified at parasite density of 47 parasites per microlitre. The presence of Borrelia was also observed, concluding to malaria co-infection with borreliosis.

Conclusions: Signs of malaria can overlap with signs of borreliosis leading to the misdiagnosis of the latter. Thick and thin smear or QBC test or molecular method may be helpful to detect both Plamodium species and Borrelia. In addition, there is a real need to consider co-infections with other endemics pathogens when diagnosing malaria.
\end{abstract}

Keywords: Malaria, Borrelia, Fever, Diagnostic, Co-infection

\section{Background}

In Senegal, several areas are endemic for tick-borne relapsing fever (TBRF) and the causal agent is Borrelia crocidurae, which is transmitted by the tick, Ornithodorus sonrai $[1,2]$. The geographic distribution of TBRF is linked to drier climates [2,3]. However, in Senegal, important extension towards the south due to climate change was observed $[1,3]$, and both malaria and tickborne relapsing fever (TBRF) due to B. crocidurae are endemic [4]. When there is no clinical evidence of other disease, falciparum malaria is the first suspected cause

\footnotetext{
*Correspondence: mamadoualpha.diallo@ucad.edu.sn

${ }^{1}$ Laboratoire de Parasitologie-Mycologie, Hôpital Aristide Le Dantec, Université Cheikh Anta Diop de Dakar, Avenue Cheikh Anta Diop, Fann, BP 5005, Dakar, Senegal

Full list of author information is available at the end of the article
}

of fever in Senegal. However, borreliosis is also a major cause of fever in Senegal [5, 6]. Thus, co-infections of malaria-borreliosis are expected to be high but are rarely reported. A patient diagnosed for malaria and TBRF is discussed.

\section{Case presentation}

A 28-year-old-male patient presented on June 9, 2016 to the health post of Pikine (sub-urban of Dakar) for headache, vomiting, diarrhea, low back pain and arthralgia. Suspecting malaria, an HRP2-based RDT specific to Plasmodium falciparum was performed. The test was positive for falciparum malaria. Following the Senegalese national policy guidelines for the treatment for uncomplicated malaria, the patient was given artemether $20 \mathrm{mg}+$ lumefantrine $120 \mathrm{mg}$ at admission and every $8 \mathrm{~h}$ for a total 
of six doses. Four days later, the patient was referred to Aristide Le Dantec Hospital. He presented with fever $\left(39^{\circ} \mathrm{C}\right)$, soreness, abdominal pain and vomiting.

The onset of symptoms had occurred brutally 4 days before, marked by intense and not radiating back pain without triggers or calming factors. There was also continuous soreness and joint pain without any particular pattern, associating intense asthenia, which is temporarily relieved by usual analgesics. There was also diffuse headache, without axial stiffness. There was no photophobia nor phonophobia, associating with vomiting and nausea, two to three fluids diarrhoeas that lasted 3 days without fever nor concept of eating suspected food and no similar cases in the entourage.

Investigations shown that the patient was from Velingara (south of Senegal) and had come to Dakar 2 months earlier. He had no previous medical history. The blood pressure was $120 / 80 \mathrm{mmHg}$. There was no sign of clinical anaemia. The rest of the examination was normal. Laboratory test showed thrombocytopenia (platelets $40 \times 10^{9} / \mathrm{mm}^{3}$ ) as the only abnormal haematological test. A thick film from peripheral blood was performed and sent to the parasitology laboratory of Le Dantec Hospital. Thick film was stained with $10 \%$ Giemsa. Trophozoite of $P$. falciparum was identified at parasite density of 47 parasites per microlitre. In addition, spirochetes of Borrelia were observed indicating a malaria co-infection with borreliosis (Fig. 1).

Treatment consisted of doxycycline $200 \mathrm{mg}$ daily during 14 days. No further symptoms were noted. Effectiveness of treatment was confirmed by negative microscopy for both malaria parasites and spirochetes.

\section{Discussion}

Malaria co-infection with borreliosis, rarely reported, poses diagnostic challenge $[4,7,8]$.

Tropical borreliosis is often disregarded [9] and is often confused with malaria, which affects the same people and presents with similar symptoms [5]. The disease called relapsing fever was considered rare until the late 1980s, before it was demonstrated that in many rural areas of Senegal, tick-borne borreliosis was the second cause of febrile diagnosis (after malaria) [1]. With an average annual incidence of $11 \%$ for over 14 years, this is the highest rate measured in Africa for a bacterial disease [10].

Borreliosis is a major public health problem in most rural areas of Senegal. However, although it is the most common bacterial disease, it remains relatively unknown by the healthcare personnel. Most likely, misdiagnosed co-infections are believed to be malaria resistant to antimalarial drugs [5]. Also, malaria is often diagnosed on the basis of only clinical symptoms and positive RDT, not microscopic examination of thick and thin smear, which

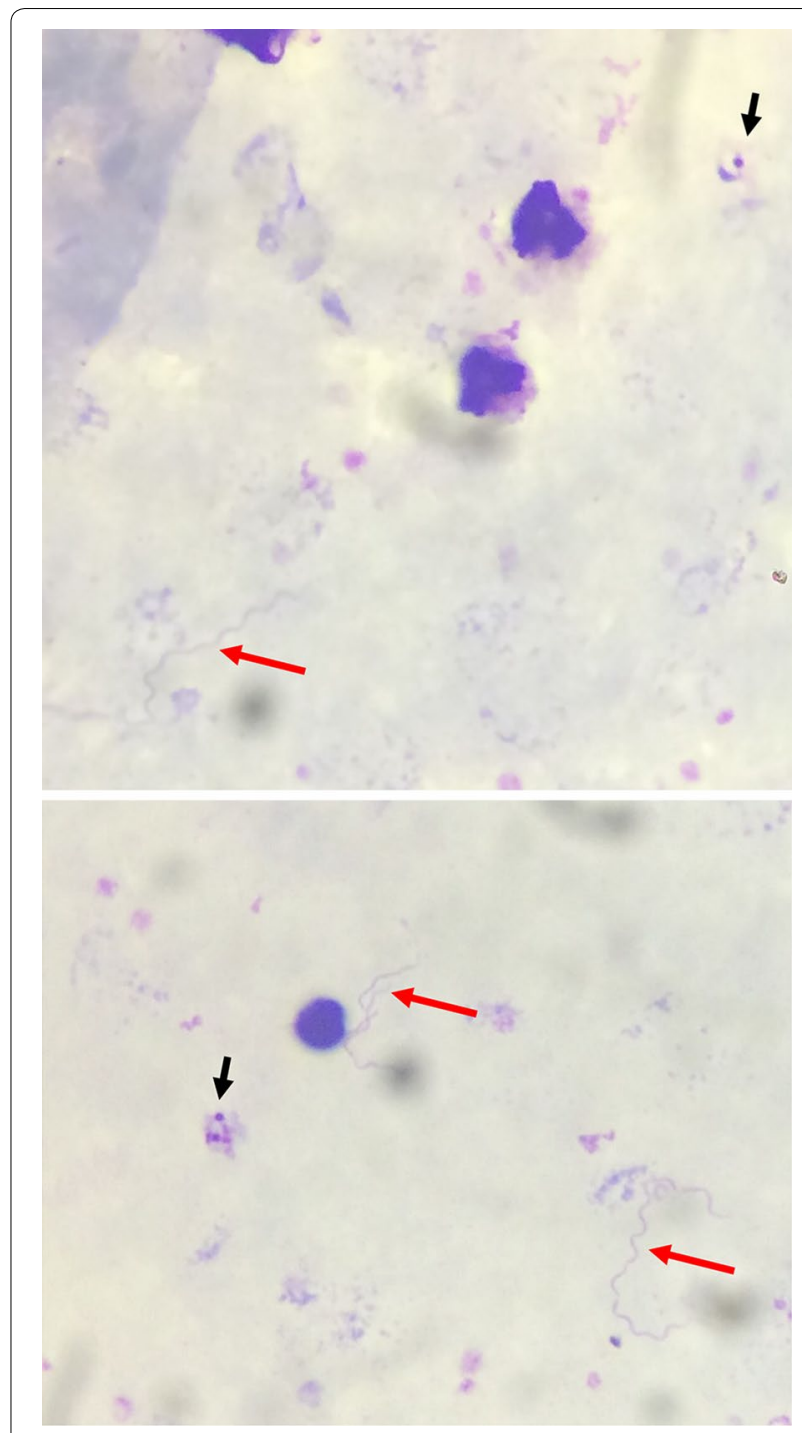

Fig. 1 Giemsa-stained thick blood smears showing trophozoite of $P$. falciparum (black arrow) and spirochetes of Borrelia (red arrow)

increases misdiagnosis of possible co-infections. In addition, B. crocidurae is present in the blood in small quantities. It is detectable in the blood samples by a trained microscopist and only during peaks of fever [6]. In the coinfection Borrelia-Plasmodium, when examining thin or thick smears, high density Plasmodium may obscure the presence of the spirochetes, making it difficult to detect the co-infecting bacteria [4]. In this case, conversely, low parasite density of $P$. falciparum was observed probably due to parasite clearance after effective treatment just after the patient was diagnosed by malaria RDT.

Routinely, the diagnosis of both tick-borne relapsing fever and malaria is based on the detection of the pathogen in the patient's peripheral blood. Borrelia and malaria parasites can be observed in Giemsa-stained thick/thin smears or fluorescence microscopy after 
centrifugation of blood in capillary tubes and staining with acridine orange (quantitative buffy coat [QBC] analysis) [3]. Borrelia can also be observed in dark field or phase contrast microscopy [3]. Positivity thresholds of thin and thick smear blood are respectively estimated at $10^{5}$ and $10^{4}$ spirochetes per milliliter of blood [4]. Giemsa method has sensitivity estimated at $25 \%$ of the reference technique (the intraperitoneal inoculation of mice is the gold standard in the diagnosis of TBRF) $[6,11]$. Long and careful examination of a thick smear made at a feverish peak achieves the sensitivity from 50 to $70 \%$.

Quantitative Buffy Coat (QBC test) is a technique for rapid detection of malaria parasites with good sensitivity and specificity. Also the QBC is very useful in Borrelia detection, allowing the technician to examine a larger volume of blood. With a positivity threshold of $10^{3} \mathrm{spi}-$ rochetes $/ \mathrm{ml}$, this technique is 100 times more sensitive than the blood smear in the diagnosis of borreliosis [4].

Velingara is out of the distribution area of tick-borne borreliosis in Senegal [2]. Thus, the patient described here was most probably infected in Pikine (Dakar), where malaria is endemic and where $O$. sonrai is abundant in burrows of mice and rats [2]. Transmission of borreliosis is well known to occur in all regions of Senegal north of the Gambian border [2, 5], explaining the high probability for people to be co-infected by Plasmodium and spirochetes. Moreover, the patient stayed in Dakar for 2 months that largely exceeds the incubation period (4-14 days) for relapsing fevers [12].

Since RDTs are provided to all health clinics in Senegal, any positive test is systematically treated as malaria without considering possible co-infections. Incorrect treatment may result in persisting symptoms leading the physician to wrongly conclude drug resistant malaria. Prompt diagnosis of TBRF and appropriate treatment would avoid developing complications such as severe meningoencephalitis [3]. The recommended treatment for borreliosis is doxycycline $100 \mathrm{mg}$ twice daily or erythromycin $500 \mathrm{mg}$ every $6 \mathrm{~h}$ per os [7].

\section{Conclusions}

This case illustrates the importance of effective and accurate diagnostic testing in tropical area where co-infections are expected to be high. TBRF should be considered in the diagnosis of every febrile patient returning from endemic areas even when malaria RDT is positive. Diagnosis relies upon routinely microscopic examination of thick and thin smear. QBC might increase the sensitivity and when available, molecular tools are highly effective in detecting spirochetes.

\section{Authors' contributions}

BSK and MD conducted clinical examination and treatment intervention. $M A D, M N$ and KD performed the microscopy examination. MAD drafted the manuscript; $A S B, K D, M N$ and $D N$ reviewed the manuscript. All authors read and approved the final manuscript.

\section{Author details}

${ }^{1}$ Laboratoire de Parasitologie-Mycologie, Hôpital Aristide Le Dantec, Université Cheikh Anta Diop de Dakar, Avenue Cheikh Anta Diop, Fann, BP 5005, Dakar, Senegal. ${ }^{2}$ Service de Médecine Interne, Hôpital Aristide Le Dantec, Université Cheikh Anta Diop de Dakar, Avenue Cheikh Anta Diop, Fann, BP 5005, Dakar, Senegal.

\section{Acknowledgements}

We thank Naomi W. Lucchi for the English reviewing of this manuscript. We thank also the Internal Medicine staff.

\section{Competing interests}

The authors declare that they have no competing interests.

\section{Availability of data and materials}

The datasets supporting the conclusions of this article are included within the article.

\section{Consent for publication}

Written informed consent was obtained from the patient for publication of this case report.

\section{Ethics approval and consent to participate}

The study received ethical clearance from the Ethic Committee of the Senegalese Ministry of Health. Informed consent to participate was obtained from the patient.

Received: 28 October 2016 Accepted: 5 January 2017

Published online: 11 January 2017

\section{References}

1. Trape JF, Godeluck B, Diatta G, Rogier C, Legros F, Albergel J, et al. The spread of tick-borne borreliosis in West Africa and its relationship to subSaharan drought. Am J Trop Med Hyg. 1996;54:289-93.

2. Trape JF, Diatta G, Arnathau C, Bitam I, Sarih M, Belghyti D, et al. The epidemiology and geographic distribution of relapsing fever borreliosis in West and North Africa, with a review of the Ornithodoros erraticus complex (Acari: Ixodida). PLoS ONE. 2013;8:e78473.

3. La Trape J. fièvre récurrente à tiques d'Afrique de l'Ouest. Ann Biol Clin. 2003;61:541-8.

4. Hovette P, Aubron C, Perrier-Gros-Claude JD, Schieman R, N'Dir MC Camara P. Value of Quantitative Buffy Coat (QBC) in borreliasis-malaria co-infection. Med Trop (Mars). 2001;61:196-7.

5. Brahim H, Perrier-Gros-Claude JD, Postic D, Baranton G, Jambou R. Identifying relapsing fever Borrelia, Senegal. Emerg Infect Dis. 2005;11:474-5.

6. Van Dam AP, Van Gool T, Wetsteyn JCFM, Dankert J. Tick-borne relapsing fever imported from West Africa: diagnosis by quantitative buffy coat analysis and in vitro culture of Borrelia crocidurae. J Clin Microbiol. 1999;37:2027-30.

7. Flatau E, Reichman N, Elias M, Raz R. Malaria and Borrelia co-infection. J Travel Med. 2000;7:98-9.

8. Miron D, Olshinky A, Assy N, Zuker M, Efrat M, Hussein O. Plasmodium and Borrelia co-infection. J Travel Med. 2004;11:115-6.

9. Mediannikov O, Socolovschi C, Bassene H, Diatta G, Ratmanov P, Fenollar F. Borrelia crocidurae infection in acutely febrile patients, Senegal. Emerg Infect Dis. 2014;20:2008-11.

10. Vial L, Diatta G, Tall A, Hadj Ba E, Bouganali H, Durand P, et al. Incidence of tick-borne relapsing fever in west Africa: Iongitudinal study. Lancet. 2006;368:37-43.

11. Diatta G, Trape JF, Legros F, Rogier C, Duplantier JM. A comparative study of three methods of detection of Borrelia crocidurae in wild rodents in Senegal. Trans R Soc Trop Med Hyg. 1994;88:423-4.

12. Rebaudet S, Parola P. Epidemiology of relapsing fever borreliosis in Europe. FEMS Immunol Med Microbiol. 2006;48:11-5. 